\title{
Automated Analysis of the Mitotic Phases of Human Cells in 3D Fluorescence Microscopy Image Sequences
}

\author{
Nathalie Harder ${ }^{1}$, Felipe Mora-Bermúdez ${ }^{2}$, William J. Godinez ${ }^{1}$, \\ Jan Ellenberg ${ }^{2}$, Roland Eils ${ }^{1}$, and Karl Rohr ${ }^{1}$
}

${ }^{1}$ University of Heidelberg, IPMB, and DKFZ Heidelberg, Dept. Bioinformatics and Functional Genomics, Im Neuenheimer Feld 364, D-69120 Heidelberg, Germany

${ }^{2}$ European Molecular Biology Laboratory (EMBL), Gene Expression and Cell Biology/Biophysics Programmes, Meyerhofstrasse 1, D-69117 Heidelberg, Germany n.harder@dkfz-heidelberg.de

\begin{abstract}
The evaluation of fluorescence microscopy images acquired in high-throughput cell phenotype screens constitutes a substantial bottleneck and motivates the development of automated image analysis methods. Here we introduce a computational scheme to process 3D multicell time-lapse images as they are produced in large-scale RNAi experiments. We describe an approach to automatically segment, track, and classify cell nuclei into different mitotic phases. This enables automated analysis of the duration of single phases of the cell life cycle and thus the identification of cell cultures that show an abnormal mitotic behavior. Our scheme proves a high accuracy, suggesting a promising future for automating the evaluation of high-throughput experiments.
\end{abstract}

\section{Introduction}

In recent years the technology of $R N A$ interference (RNAi) has become the method of choice for identifying the biological function of genes in the field of functional genomics. With this screening method all known genes of an organism are systematically silenced one after the other, and the resulting morphological changes are analyzed. However, such large-scale knockdown screens provide enormous amounts of data which require tools for automated image analysis.

Our work is carried out within the EU project MitoCheck, which aims to explore the coordination of mitotic processes in human cells at a molecular level and to contribute to revealing the mechanisms of cancer development. To identify the genes that are involved in cell division (mitosis), genome-wide high-throughput RNAi primary screens are performed. In addition, RNAi secondary screens are performed which have a higher spatial and temporal resolution and involve several hundred genes. Fluorescence microscopy time-lapse images of the treated cell culture are acquired to study the effect of a silenced gene on mitosis. This contribution is concerned with the automated evaluation of an assay to analyze the duration of the different phases in mitotic progression. In normal cells of one type the duration of the mitotic phases is similar. To study the duration of mitotic phases of treated cells a specific assay has been developed which is 
characterized by significant elongations of mitotic phases compared to control experiments. To automatically determine whether mitotic phases of treated cells are longer compared to normal cells, cells have to be observed throughout their life cycle and for each time point the respective phase has to be determined.

Previous work on automated analysis of cell images has been done in different application fields. Based on fluorescence microscopy imaging complete cells as well as single subcellular structures have been studied. The automated recognition of subcellular structures is a major task in location proteomics and work has been done in this field given 2D (e.g., [1,2]) and 3D (e.g., 3]) single cell images. Classification of complete cells has been performed, for example, to investigate the influence of drugs on cellular proteins [4. In [5] tracking of cells in 2D microscopy images has been used to improve segmentation and cell quantification. Automated analysis of cell images plays an increasing role particularly for the evaluation of high-throughput cell phenotype screens. Approaches for single-frame multi-cell $2 D$ images have been described in $6,7,8$.

We have developed an approach to analyze multi-cell image sequences from large-scale RNAi secondary screens. In comparison to previous work, we analyze $3 D$ cell array time-lapse images that include multiple cell nuclei in different mitotic phases. Each image of an image sequence contains three slices that have been acquired with a confocal fluorescence microscope. Our computational scheme for analyzing the duration of mitotic phases consists of four main steps: Segmentation of multi-cell images, tracking of cells, image feature extraction, and classification into the mitotic phases. For fast and accurate segmentation we use a region adaptive thresholding technique. Our tracking scheme is able to cope with the splitting of cells during mitosis which is important in our application. Based on the tracking result we automatically select the most informative slice out of the 3D image, which is then used for feature extraction. Compared to previous work, we incorporate temporal changes of the cell morphology between ancestrally related cells by directly including dynamic image features. A Support Vector Machine classifier is used to classify the cells into the following seven mitotic phases: Interphase, Prophase, Prometaphase, Metaphase, Anaphase1, Anaphase2, and Telophase. Based on the classification result in subsequent images the duration of the different phases can be determined. It is the first time that an automated scheme is presented which yields a detailed classification into the above mentioned phases of dividing cells. Our approach has been successfully applied to four image sequences from large-scale RNAi screens. We have compared the performance with ground truth provided by manual evaluation and it turned out that we obtain an average accuracy of around $89 \%$ and an overall accuracy of $94.6 \%$ for the classification.

\section{Methods}

\subsection{Image Analysis Workflow}

For a detailed analysis of the different phases of dividing cells high-resolution confocal fluorescence microscopy images of the DNA have been acquired. Compared 


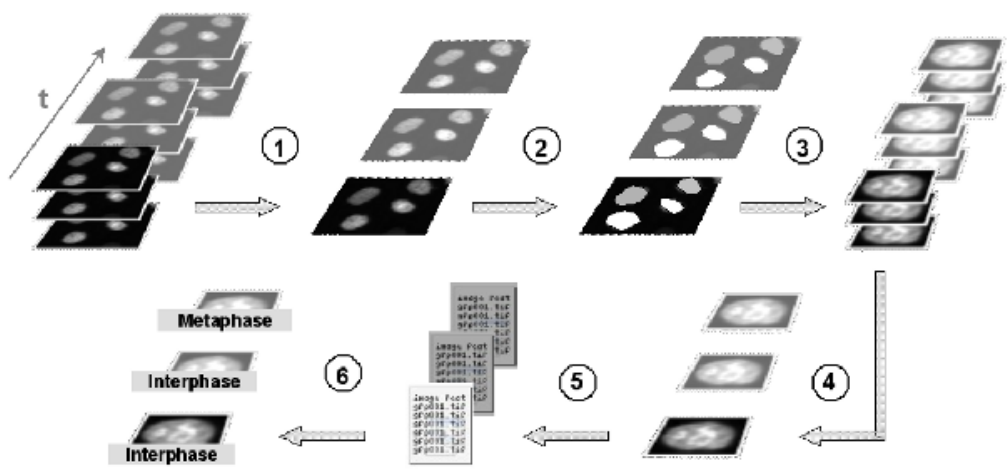

Fig. 1. Image analysis workflow: (1) Maximum intensity projection of multi-cell 3D images, (2) Segmentation and tracking in 2D, (3) Extraction of 3D ROIs including single cells, (4) Selection of most informative slice, (5) Feature extraction, (6) Classification

to previous work we here use 3D images consisting of three confocal planes (slices). The reason to use several confocal planes is that cells change their morphology during mitosis, i.e., in the interphase they are flat, but with the beginning of cell division they arch upward and take the shape of a hemisphere. Therefore, if we would use the same focal plane throughout the cell cycle the cell structure (DNA) could not well be observed. Since we are using multi-cell images that contain cells in different mitotic phases, it is impossible to define one focal plane per time step that well represents all cells. Therefore, three slices from three different focal planes are acquired that cover the range of possible cell shape changes. Because of technical reasons in the image acquisition process the number of slices is restricted to three. This relatively small number of slices does not allow a complete 3D analysis. Thus, we apply the workflow shown in Fig. 1, In the first step, we apply a maximum intensity projection (MIP) for each time step resulting in $2 \mathrm{D}$ images. In the second step, we perform segmentation and tracking based on these MIP images to determine the correspondences of cell nuclei in subsequent frames. Based on the segmentation and tracking result we now go back to the original 3D images and define 3D ROIs for each cell. For each 3D ROI we choose the slice containing the most information. The reason of using the original images lies in the fact that these images contain more detailed information about the inner intensity structure of a cell than the MIP images. Finally, static and dynamic features are extracted and classification is performed resulting in a sequence of mitotic phases for each cell trajectory.

\subsection{Segmentation of Multi-cell Images}

Since we have to cope with a huge number of multi-cell images, fast and reliable segmentation of single objects is crucial. Various advanced segmentation algorithms have been described in the literature, but as computation time plays an important role in our application, the speed of the algorithm is a decisive criterion. We have investigated three different thresholding techniques which we 
(a)

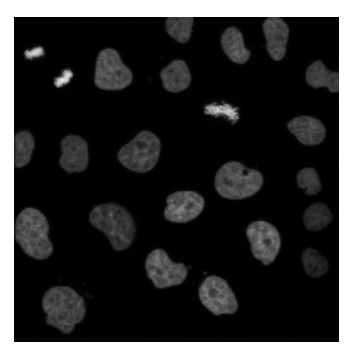

(b)

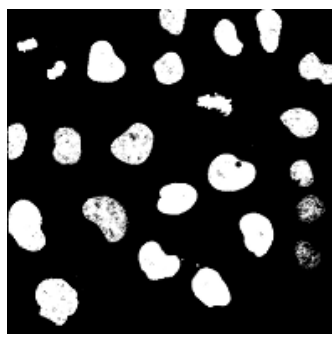

(c)

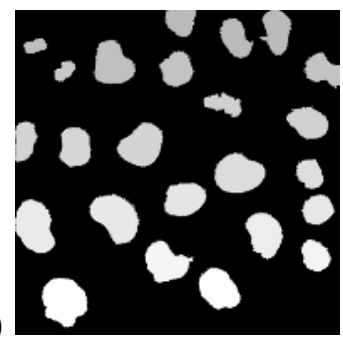

Fig. 2. (a) Original image, (b) Global threshold (Otsu), (c) Local adaptive threshold

have previously used for analyzing static $2 \mathrm{D}$ multi-cell images. It turned out that global thresholding alone does not produce satisfying results especially for low-contrast cells. Instead, local adaptive thresholding can segment the cell nuclei with high accuracy (see Fig. 2). Consequently, we apply the latter technique which incorporates several improvements compared to [8]. The algorithm uses a quadratic sliding window to calculate local thresholds for different image regions. A local threshold is only calculated if the variance within the window reaches a user-defined threshold, else a global threshold is used 9]. This ensures that only for regions that contain some information the local threshold is calculated, which significantly reduces the computation time. The threshold is applied to a quadratic region at the center of the sliding window and the window is shifted by the length of this inner region. Compared to [8] this modification reduces segmentation errors at the region borders. To calculate the global and the local intensity thresholds we applied two histogram-based threshold selection schemes: Otsu thresholding [10] and minimum error thresholding [11. We found that the combination of [10] to determine the global threshold and [11] to determine the local thresholds yielded the best results (see Fig. 25).

\subsection{Tracking of Mitotic Cell Nuclei}

To analyze the mitotic behavior of single cells in multi-cell images, a tracking scheme is required that determines the temporal connections and can handle splitting objects. We have developed the following two-step tracking scheme: First, initial, non-splitting trajectories are established, and second, mitotic events are detected and the related trajectories are merged. In the first step, the initial trajectories are determined using a feature point tracking algorithm based on [12. As feature points we use the centers of gravity of segmented cell nuclei. For each frame of an image sequence the algorithm considers the predecessor and successor frame. In these frames, object correspondences are determined by searching for trajectories with maximum smoothness. In the second step, all trajectories that do not start in the first frame are taken into account as possible mitotic events. The decision whether a trajectory should be treated as mitosis event is made depending on the distance-overlap-ratio of potential parent and child objects [13. In addition, the smoothness of the potential compound trajectory is taken into account. For the identified splitting events the corresponding tracks are finally merged. As an example, Fig. 3 shows a tracking result. 
(a)

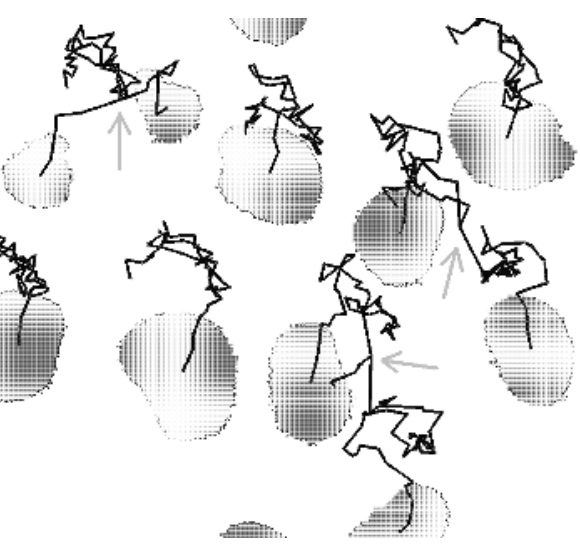

(b)
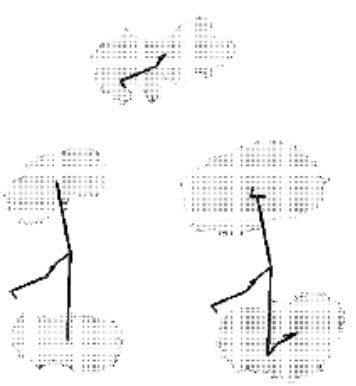

Fig. 3. (a) Tracking result including three mitotic cells, (b) Example of the splitting of one cell in three consecutive images (top, bottom left, bottom right)

\subsection{Extraction of Static and Dynamic Features}

As described in Section 2.1, we determine for each cell nucleus 3D ROIs in the original 3D images. Within these ROIs we select the cell's individual most informative slice in order to compute image features. Two selection criteria for determining the optimal slice have been tested: Maximum total intensity and maximum entropy. We found that the maximum-intensity criterion performs very well compared to manual selection, whereas the maximum-entropy criterion often fails and shows a high sensitivity to noise. Consequently, we here apply the maximum-intensity criterion. Within the selected slice we compute a set of static and dynamic image features. The static features comprise granularity features, object- and edge-related features, tree-structured wavelet features, Haralick texture features, grey scale invariants, and Zernike moments. The dynamic features represent the morphological changes of cells in adjacent frames. To this end, we compute the difference of three basic image features (object size, mean intensity, and standard deviation of the intensity) for each cell to its predecessor and to its successor. We chose these three features because they exhibit characteristic changes during mitosis. As an example, Fig. [4 shows a plot of the mean intensity over time for a dividing cell. It can be seen that, for example, between Prophase and Prometaphase there is a steep rise of the mean intensity and for Anaphase1 there is a characteristic spike. In total, we compute 332 features for each cell nucleus. For the training set, we standardize each feature w.r.t. a mean value of zero and a standard deviation of one. In the test set, the feature values are linearly transformed based on the transformation parameters of the training set.

\subsection{Classification}

We apply a Support Vector Machine (SVM) classifier with a Radial Basis Function (RBF) as kernel function to classify the nuclei into the seven classes Interphase, Prophase, Prometaphase, Metaphase, Anaphase1, Anaphase2, and 


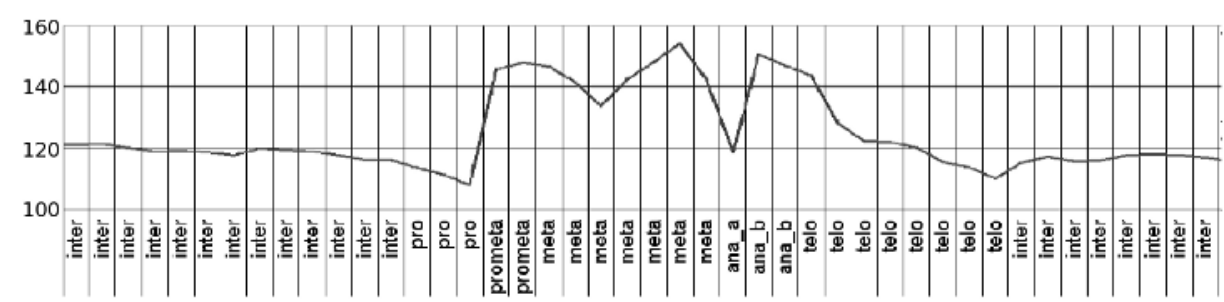

Fig. 4. Mean intensity for a dividing cell and manually assigned phases of mitosis

Telo-phase (see Fig. 5). SVMs are mathematically well-founded and they have the advantage that their complexity is independent of the dimension in feature space compared to other classification methods. This property allows us to work with a high number of features and we can skip the feature selection step as it is not crucial. We solve the multi-class classification problem with a "one-againstone" approach. To optimize the penalty parameter $\mathrm{C}$ and the kernel parameter $\gamma$ for the Radial Basis Function, we perform a three-fold cross-validation with varying values of $\mathrm{C}$ and $\gamma$ on the training set (model selection) prior to the actual training of the classifier.

\section{Experimental Results}

\subsection{Image Data}

In our study we use 3D image sequences that have been acquired with a confocal fluorescence microscope in the framework of the EU project MitoCheck at the EMBL Heidelberg. Chromosome morphology is visualized using a HeLa (Kyoto) cell line stably expressing the chromosomal marker histone 2B-EGFP. One image contains about 20 nuclei with an average diameter of approximately 100-150 pixels (in Interphase). All images have a gray value depth of 8 bit and a spatial resolution of $1024 \times 1024$ pixels. Each image sequence comprises 124 to 128 time steps with a temporal resolution of $7 \mathrm{~min}$ and three image slices per time step.

\subsection{Classification Results}

We have applied our approach given four 3D multi-cell image sequences. Using maximum intensity projection for all stacks of all sequences resulted in 500

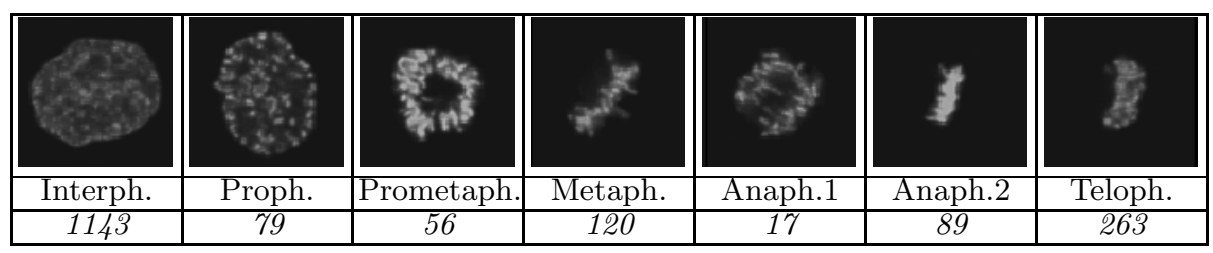

Fig. 5. Example images of the different mitotic phases and number of available samples 
Table 1. Confusion matrix and accuracies for the experiment with five-fold cross validation; dynamic features have not been included; overall accuracy: $92.9 \%$

\begin{tabular}{|c||c|c|c|c|c|c|c|c|}
\hline \multirow{2}{*}{$\begin{array}{c}\text { True } \\
\text { Class }\end{array}$} & \multicolumn{9}{|c|}{ Classifier Output } & $\begin{array}{c}\text { Accur. } \\
{[\%]}\end{array}$ \\
\hline \hline Inter & $\mathbf{1 1 0 9}$ & 8 & 0 & 1 & 0 & 0 & 26 & 97.0 \\
\hline Pro & 11 & $\mathbf{6 6}$ & 1 & 0 & 1 & 0 & 0 & 83.5 \\
\hline Prometa & 0 & 0 & $\mathbf{4 4}$ & 6 & 6 & 0 & 0 & 78.6 \\
\hline Meta & 1 & 0 & 5 & $\mathbf{1 1 0}$ & 0 & 0 & 4 & 91.7 \\
\hline Ana1 & 1 & 0 & 5 & 3 & $\mathbf{5}$ & 2 & 1 & 29.4 \\
\hline Ana2 & 0 & 0 & 0 & 0 & 1 & $\mathbf{7 6}$ & 12 & 85.4 \\
\hline Telo & 21 & 0 & 0 & 1 & 2 & 8 & $\mathbf{2 3 1}$ & 87.8 \\
\hline
\end{tabular}

Table 2. Same as Tab. 1 but including dynamic features; overall accuracy: $94.6 \%$

\begin{tabular}{|c||c|c|c|c|c|c|c|c|}
\hline \multirow{2}{*}{$\begin{array}{c}\text { True } \\
\text { Class }\end{array}$} & Inter & Pro & Prometa & Meta & Ana1 & Ana2 & Telo & $\begin{array}{c}\text { Accur. } \\
{[\%]}\end{array}$ \\
\hline \hline Inter & $\mathbf{1 1 1 4}$ & 6 & 0 & 1 & 0 & 0 & 22 & 97.5 \\
\hline Pro & 5 & $\mathbf{7 2}$ & 2 & 0 & 0 & 0 & 0 & 91.1 \\
\hline Prometa & 0 & 0 & $\mathbf{5 1}$ & 5 & 0 & 0 & 0 & 91.1 \\
\hline Meta & 0 & 0 & 2 & $\mathbf{1 1 4}$ & 1 & 1 & 2 & 95.0 \\
\hline Ana1 & 1 & 0 & 2 & 2 & $\mathbf{1 1}$ & 1 & 0 & 64.7 \\
\hline Ana2 & 0 & 0 & 0 & 0 & 1 & $\mathbf{8 4}$ & 4 & 94.4 \\
\hline Telo & 33 & 0 & 0 & 1 & 1 & 3 & $\mathbf{2 2 5}$ & 85.6 \\
\hline
\end{tabular}

projected multi-cell images. These images have been segmented and tracked. The tracking scheme was able to detect $80.0 \%$ of all occurring mitosis events (determined by visual inspection). Since the subsequent processing steps rely on correctly detected mitosis events we corrected the remaining ones manually. Static and dynamic features have been extracted within the most informative slices for all segmented and tracked nuclei. To provide ground truth, all nuclei have been classified manually into the seven phases of mitosis. We split the available samples for each class randomly into training data and test data at a ratio of 2:1. The numbers of samples and example images for each class are given in Fig. 5. The feature values were standardized and a Support Vector Machine classifier was trained as described above. For the different phases (classes) we obtain between $80.0 \%$ and $98.1 \%$ correct classifications and an average accuracy of $90.1 \%$ (data not shown). To check the reliability of the result we repeated the classification step, applying a five-fold outer cross-validation on the whole data set. As shown in Tab. 2, we obtain classification accuracies of $64.7 \%$ to $97.5 \%$ for the different phases and an average accuracy of $88.5 \%$. Thus, both average accuracies correspond well and we can draw the conclusion that we can rely on an average classification accuracy of around $89 \%$. If we do not just average the accuracies for the different classes but take into account the number of samples per class we obtain an overall accuracy of $94.6 \%$.

To examine the effect of including dynamic features, we removed all dynamic features and repeated the classification experiments. In this case, we obtained an average accuracy of $79.1 \%$ and an overall accuracy of $92.9 \%$ for the experiment 
with five-fold cross-validation (see Tab.10). It turns out that by including dynamic features we can improve the result significantly. The improvement becomes even more obvious when we compare the accuracies for the single phases of both experiments as listed in Tabs. 1 and 2, If dynamic features are included the accuracies are significantly higher for all phases, except for Telophase. The largest improvement can be observed for Anaphase1 with an increase from $29.4 \%$ to $64.7 \%$ and for Prometaphase with an increase from $78.6 \%$ to $91.1 \%$.

\section{Discussion and Conclusion}

We have presented an approach for automated analysis of the duration of mitotic phases in 3D confocal microscopy image sequences. Our approach segments and tracks splitting cells throughout the sequences and thus determines treestructured tracks which represent cell pedigrees. By using static and dynamic features our scheme classifies the cells with high accuracy into seven mitotic phases. Based on the sequence of phases that have been computed in subsequent frames, the duration of each phase can be determined. The computed phase durations can be compared to control experiments to automatically identify experiments that show delays in mitotic phases.

\section{Acknowledgement}

This work has been supported by the EU project MitoCheck.

\section{References}

1. Huang, K., Murphy, R.: Boosting accuracy of automated classification of fluorescence microscope images for location proteomics. BMC Bioinf. 5 (2004) 78

2. Conrad, C., Erfle, H., Warnat, P., Daigle, N., Löch, T., Ellenberg, J., Pepperkok, R., Eils, R.: Automatic identification of subcellular phenotypes on human cell arrays. Genome Research 14 (2004) 1130-1136

3. Chen, X., Murphy, R.: Robust classification of subcellular location patterns in high resolution 3D fluorescence microscope images. In: Proc. 26th Int. Conf. IEEE Engineering in Medicine and Biology Society, San Francisco, CA (2004) 1632-1635

4. Lindblad, J., Wählby, C., Bengtsson, E., Zaltsman, A.: Image analysis for automatic segmentation of cytoplasms and classification of Rac1 activation. Cytometry Part A 57A (2003) 22-33

5. Yang, F., Mackey, M., Ianzini, F., Gallardo, G., Sonka, M.: Cell segmentation, tracking, and mitosis detection using temporal context. In: Proc. MICCAI'2005. Volume 3749., Palm Springs, CA, USA, Springer-Verlag (2005) 302-309

6. Perlman, Z., Slack, M., Feng, Y., Mitchison, T., Wu, L., Altschuler, S.: Multidimensional drug profiling by automated microscopy. Science 306 (2004) 1194-1198

7. Zhou, X., Liu, K.Y., Bradley, P., Perrimon, N., Wong, S.T.: Towards automated cellular image segmentation for RNAi genome-wide screening. In: Proc. MICCAI'2005. Volume 3749., Palm Springs, CA, USA, Springer-Verlag (2005) 302-309 
8. Harder, N., Neumann, B., Held, M., Liebel, U., Erfle, H., Ellenberg, J., Eils, R., Rohr, K.: Automated recognition of mitotic patterns in fluorescence microscopy images of human cells. In: Proc. ISBI'2006, Arlington, USA (2006) 1016-1019

9. Gonzalez, R., Woods, R.: Digital Image Processing. Prentice Hall (2002)

10. Otsu, N.: A threshold selection method from grey level histograms. IEEE Transactions on Systems, Man and Cybernetics 9 (1979) 62-66

11. Kittler, J., Illingworth, J.: Minimum error thresholding. Pattern Recognition 19 (1986) 41-47

12. Chetverikov, D., Verestoy, J.: Tracking feature points: a new algorithm. In: Proc. 14th Int. Conf. Pattern Recognition, Brisbane, Australia (1998) 1436-1438

13. Withers, J.: Tracking cell splits and merges. In: Southwest Symp. Image Analysis and Interpretation. (1996) 117-122 